\title{
NUMERICAL MODELING OF SEPARATED FLOWS IN THREE-DIMENSIONAL DIFFUSERS AND APPLICATION OF SYNTHETIC JETS FOR SEPARATION CONTROL
}

\section{S. Yu. Krasheninnikov, D. E. Pudovikov, and S. A. Torohov}

\author{
P. I. Baranov Central Institute of Aviation Motors (CIAM) \\ Aviamotornaya Str. 2, Moscow, Russia
}

\begin{abstract}
Solving the problem of creating an environmentally friendly "green plane" implies development and implementation of several actions aimed at increasing airplane performance and reducing environmental contamination. One possible way to solve this problem is to reduce the powerplant weight, in particular, by decreasing its length. The airplane engine flowpath comprises transition ducts: those between the low- and high-pressure compressors, between the compressor and combustor, and between the high- and low-pressure turbines. In a modern high-bypass turbofan, the flowpath varies in the streamwise direction. Shorter transition ducts have greater curvature. Because of this, intensive separation may occur, which leads to increased losses in the flowpath and to significant growth of nonuniformity of flow parameters. Vast experience of numerical and experimental studies of unsteady separated flows has been accumulated by now. In many cases, however, these investigations are performed in a two-dimensional (2D) formulation, which is primarily caused by the high cost of three-dimensional (3D) unsteady calculations. The numerical and experimental work [1] shows that flows in diffuser ducts can have an essentially unsteady 3D structure. This is valid even for ducts modeling $2 \mathrm{D}$ configurations. This paper describes the results of a numerical study of the flow structure and its features in model S-shaped transition ducts, as well as the results of using a synthetic jet generator for flow control and for reduction of total pressure losses. Three-dimensional flows are numerically modeled by the unsteady Reynolds-averaged Navier-Stokes (URANS) / RANS methods. The calculations show that the use of the synthetic jet generator can lead to duct loss reduction by $45 \%$.
\end{abstract}

This is an Open Access article distributed under the terms of the Creative Commons Attribution-Noncommercial License 3.0, which permits unrestricted use, distribution, and reproduction in any noncommercial medium, provided the original work is properly cited. 


\section{DESIGN FLOW STUDY IN AN ANNULAR DUCT BY THE RANS/URANS METHODS}

A diagram of the examined S-shaped duct with an annular cross section is shown in Fig. 1. Such a duct shape (with horizontal entrance and exit) is typical of transition ducts between the high- and low-pressure turbines of advanced engines and takes into account some in-process and design constraints on the geometry of flowpath elements. In addition to the curvilinear section under study, the design area also includes the initial and final constant-area rectilinear sections.

The calculation procedure for separated flows in spatial diffuser ducts was improved by an example of a duct considered in [2] and by design and experiments $[3,4]$. An in-house code was used for calculations $[1,5,6]$.

It was shown [1] that flows in the ducts considered have a complex spatial structure. The RANS calculations predict an almost periodic system of a pair of swirl plaits in an annular duct and a pair of plaits of greatly different lateral sizes in a rectangular duct.

Figures $2 a$ and $2 b$ show the results in the annular duct calculated with different circumferentially uniform meshes. The velocity fields in the curvilinear exit section are presented. The mesh dimension displays the number of cells in the circumferential and radial directions. With the mesh having 38 cells in the radial direction (see Fig. $2 a$ ), the parameter $y+$ determined on the basis of the size of the first cell near the wall is of the order of 10 everywhere on the wall, and the cell size pitch near the wall is $25 \%$. The mesh having 120 cells in the radial direction (see Fig. $2 b$ ) is adapted to the walls well enough to ensure an adequate boundary-layer resolution, i. e., $y+$ is of the order of 1 everywhere, and the cell pitch is $10 \%$.

The data presented here show that the vortex structure is not resolved by the coarse mesh. The vortex structure can be captured only by a rather fine mesh (in the example considered, the angular cell size should be smaller than $\left.3^{\circ}\right)$. The cell quality in the radial direction and the degree of boundary-layer resolution, on the contrary, turn out to be less essential factors if the law of the wall is used.

A comparison of design data with the experiment [1] shows that stationary approaches appreciably underpredict the total pressure losses in the duct.

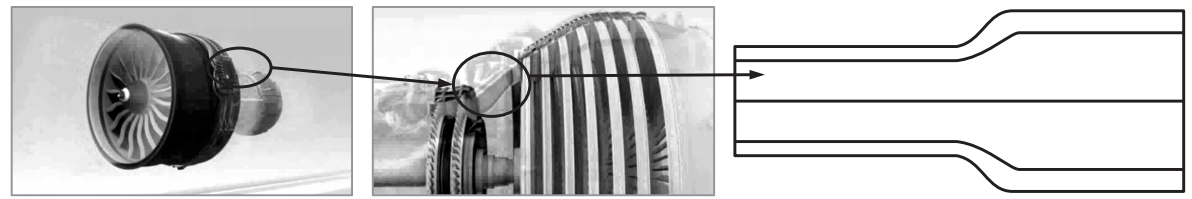

Figure 1 Diagram of the model annular transition duct 


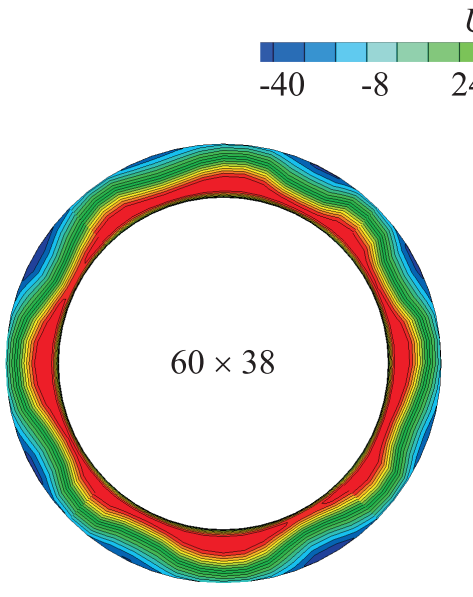

(a)

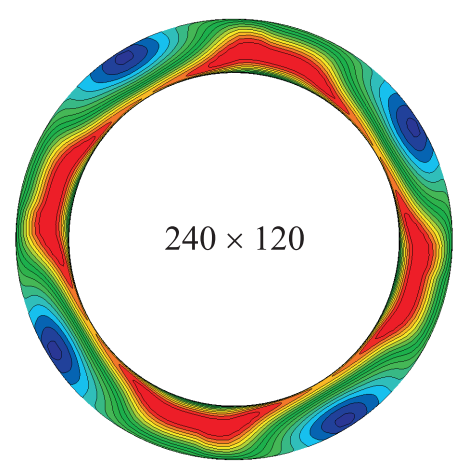

(b)

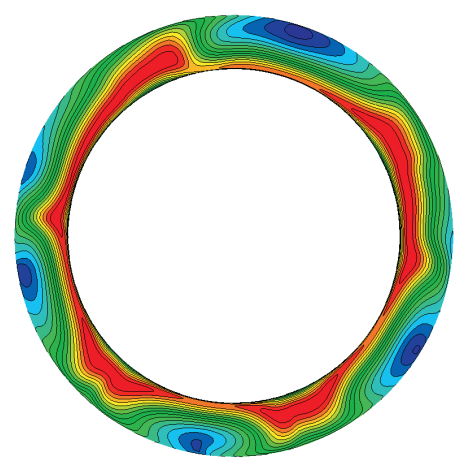

(c)

Figure 2 Mesh quality and nonuniformity of the flow structure. (Refer Krasheninnikov et al., p. 523.)

Thus, when stationary RANS methods are used for flow calculations in spatial diffusers, the accuracy of determining aerohydrodynamic parameters is rather low; in some cases, even a qualitatively correct description of the flow cannot be obtained. Probably, it is due to the essentially nonstationary and unstable nature of separated flows. The separation disintegrates into smaller structures whose scale is commensurable with the vertical size of the duct. Satisfactory accuracy of calculations is provided only by methods based on solving nonstationary Reynolds or Navier-Stokes equations. Unsteady calculations (URANS) show that the flow structure changes with time. There is unsteady circumferential motion in the annular duct and vortices of varied size in the rectangular duct. 
A further analysis shows that the structure being formed is sensitive to minor nonuniformities of the flow field. For studying the effect of circumferential nonuniformity, the flow is modeled in a duct containing bluff struts in the flowpath. The constructed mathematical model of the design area allows up to eight struts to be described simultaneously owing to modification of the boundary conditions on internal surfaces.

The flow design results obtained (see Fig. $2 a$ ) characterize the influence of circumferential nonuniformity on the flow structure. Insertion of struts into the duct complicates the flow structure. The structure obtained traces both the number of struts and their positions. This fact, as well as the small mesh nonuniformity discovered later (presence of "seams" on block edges, changing the orientation of one of the cell edges by less than $10^{-5}$ degrees), is indicative of flow sensitivity to any kind of nonuniformity, which can serve both as a generating and a stabilizing factor.

To verify this statement, a similar approach was used for series of calculations with the Fluent software package. An algebraic mesh was generated, which provided no circumferential nonuniformity within the calculation accuracy. In the problem considered through unsteady calculations, the structure obtained was circumferentially rotating. When a weak geometric nonuniformity of the inlet section was introduced, such that the axial velocity distortion at the curvilinear inlet section of the duct did not exceed $3 \%$, the flow was stabilized.

The influence of the design area size (angular scale of the design sector in the case of an annular duct) on the flow structure should be noted. At small angular scales, the vortex structure was not formed.

\section{APPLICATION OF SYNTHETIC JETS FOR SEPARATED FLOW CONTROL}

It is unacceptable in practice to use diffusers with developed flow separations because of a drastic increase of losses in the duct and intensive pressure oscillations in separation zones, which can destroy engine elements. For application of short diffusers with a large apex angle, the separation phenomenon should be eliminated or appreciably attenuated. Various flow control methods can be applied for this purpose: injection, suction, interceptors, etc. From the viewpoint of possible control, energy efficiency, and impact on flow, the most promising methods are various nonstationary control actions; among them, the use of synthetic jets should be noted. Such jets are usually generated by a cavity having one or several moving walls-membranes and communicating with the main stream through a hole.

The flow induced by an isolated generator of synthetic jets was initially extensively studied for the case of bounded space. There are experimental works $[7,8]$ 


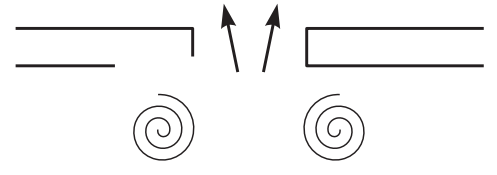

(a)

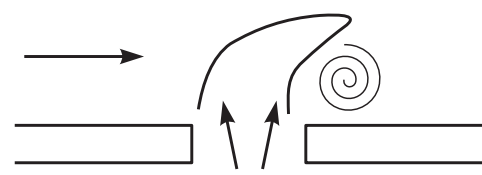

(b)

Figure 3 Jet interaction with the quiescent ambient medium $(a)$ and with an air flow moving along the plate surface $(b)$

in which the flow field, transitory processes, and vortex structure are presented in sufficient detail for verification of numerical models.

When the jet interacts with the ambient medium, the vortex motion arises on its boundary (Fig. 3a), which is the main subject of the study. If there is a ramjet, however, another effect becomes also important, namely, jet interaction with the flow and generation of vortices similar to those that appear when interceptors interact with the flow (Fig. $3 b$ ). The calculations predict an optimum value of injected jet penetration into the core flow, which approximately corresponds to an optimum interceptor height, i. e., it should be $20 \%-30 \%$ greater than the boundary-layer thickness.

Moreover, the suction tact is also affected by the boundary-layer suction effect. The efficiency of using synthetic jets for flow control in a separation diffuser depends on superposition of all these effects (i. e., on intensity of boundary-layer mixing and replacement of the low-energy gas in the boundary layer by the highenergy gas from the core flow). In turn, these effects are governed by the slot size and by the amplitude and frequency of membrane vibrations.

A model stationary problem with injection through a slot from a cavity into an air flow moving along the plate surface was solved to study the slot shape influence on mixing intensity (see Fig. $3 b$ ). The slot area, gas flow, and temperature are fixed. The varied parameter is the dimensionless slot length $(l / h$, length-to-width ratio): from 12 to $3 / 4$. Figure 4 shows the results calculated for two extreme values of $l / h$.

In the model problem considered, a jet exhausted from a short and broad slot ensures more intensive mixing with the flow. The temperature field inside the "mixing spot," where the gas temperature is lower than the flow temperature, is more uniform, and the spot size is smaller. The boundary layer is also more effectively mixed by a wide jet.

Therefore, in solving the problem of choosing optimum parameters of the synthetic jet generator, it is necessary to take into consideration not only the generator, amplitude, and frequency parameters, but also the slot shape.

In calculations with flow control by synthetic jets, a single-slot duct sector was considered. The duct geometry was similar to that considered in section 1 . The 


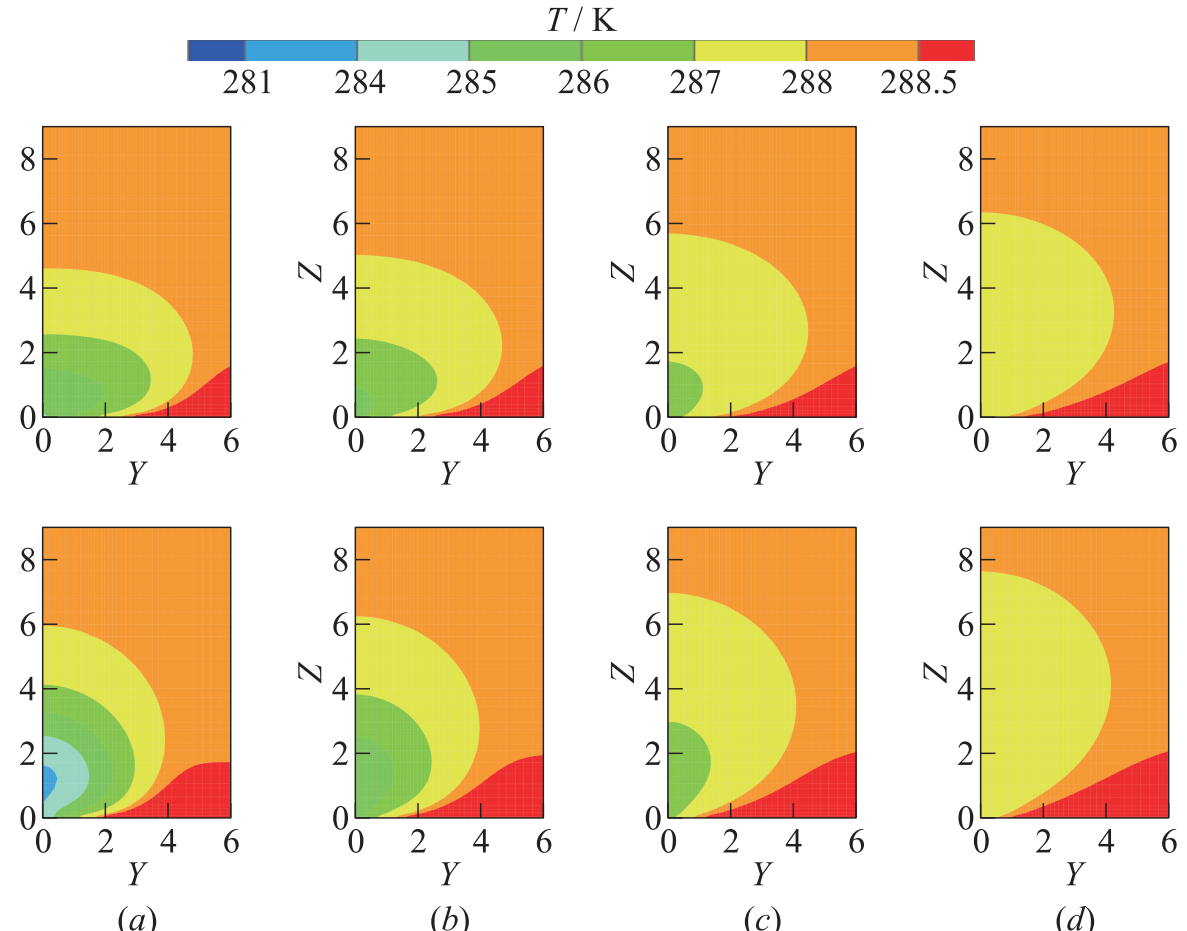

Figure 4 Temperature distribution in cross sections downstream of the jet: $x$ $=24(a), 32(b), 48(c)$, and $64 \mathrm{~m}(d) ; l / h=3 / 4$ (upper row) and 12 (lower row). (Refer Krasheninnikov et al., p. 526.)

generator was a cavity with a movable wall attached to the main duct (Fig. 5). The slot size was $0.5 \times 1 \mathrm{~mm}$; the slot was oriented along or across the flow.

The intensive separation forming in the duct is characterized by nonstationary fluctuations of parameters, which do not have a characteristic frequency [1]. If a periodic impact is imposed, the characteristic time scale in the case considered exceeds the period of membrane wall motion by a factor of 5-7. Therefore, the total time needed to calculate one variant is rather large: approximately $4000 \mathrm{~h}$ of processor time.

Figure 6 shows the calculated instantaneous distribution of axial velocity in the duct under study with the synthetic jet generator in operation. At a typical axial velocity of the core flow equal to $\sim 100 \mathrm{~m} / \mathrm{s}$ and membrane motion frequency of $1 \mathrm{kHz}$, the time needed for the gas particle to pass through the curvilinear section corresponds approximately to three control cycles, which is clearly seen in these illustrations. 


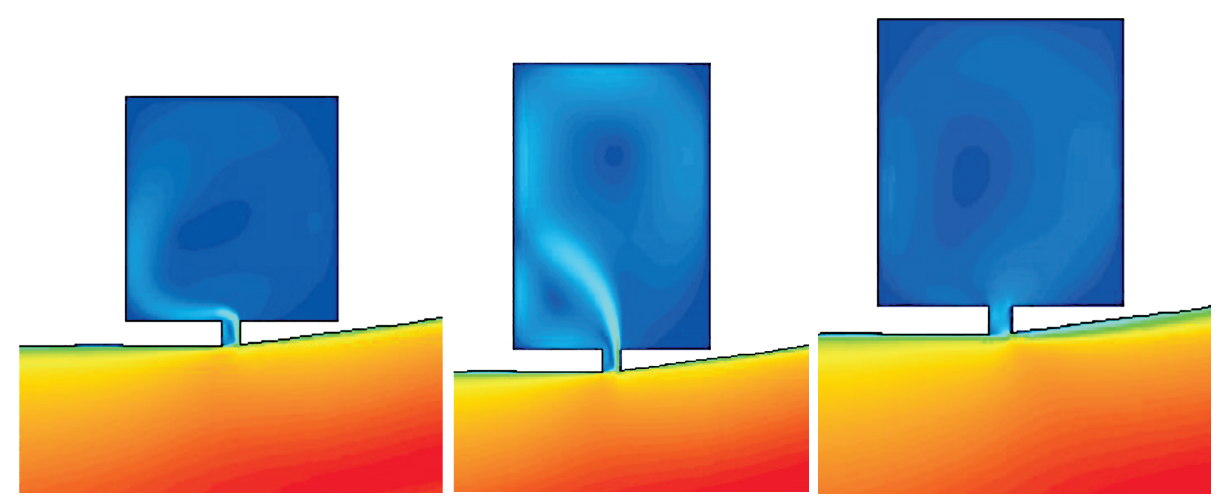

Figure 5 Different stages of operation of the synthetic jet generator. (Refer Krasheninnikov et al., p. 527.)
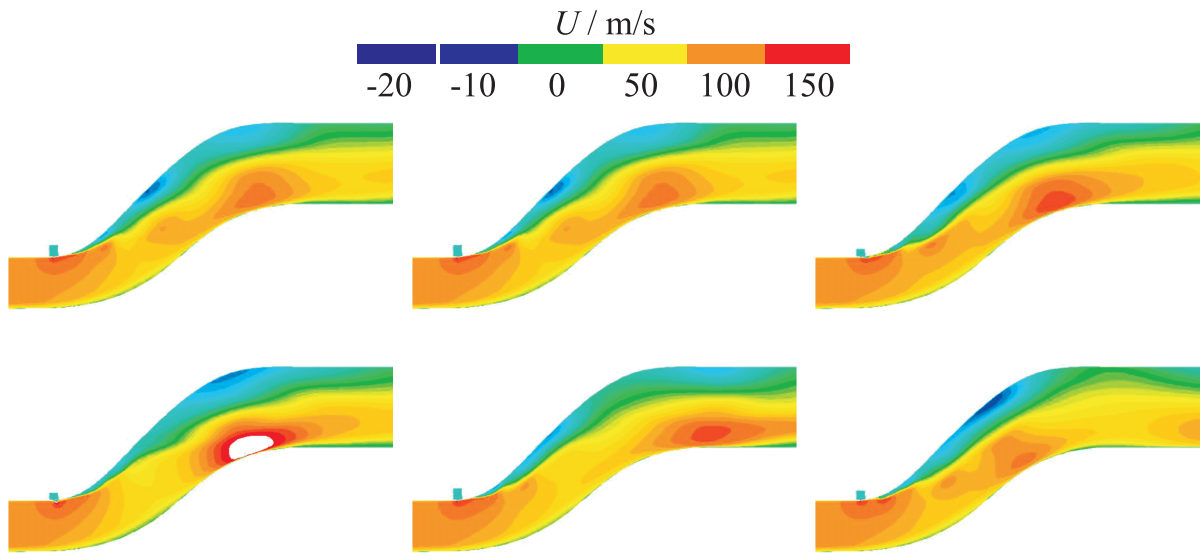

Figure 6 Instantaneous distribution of axial velocity within one operation cycle of the synthetic jet generator. (Refer Krasheninnikov et al., p. 527.)

The main goal of the study performed was to calculate the total pressure loss in the transition duct by the formula

$$
\Delta \sigma=\frac{p_{\text {in }}^{*}-p_{\text {out }}^{*}}{p_{\text {in }}^{*}}
$$

where $p_{\text {in }}^{*}$ and $p_{\text {out }}^{*}$ are the time-averaged values of the flow-averaged total pressure in the S-shaped duct input and output sections, respectively.

The values of $\Delta \sigma$ determined for fixed membrane motion amplitudes and frequencies are listed in Table 1. It is seen from these data that the total pressure 
Table 1 Results of calculations

\begin{tabular}{lc}
\hline Hole & $\Delta \sigma, \%$ \\
\hline Without & 3.15 \\
Along & 1.73 \\
Across & 1.99 \\
\hline
\end{tabular}

loss is reduced by $45 \%$, as compared with duct losses without flow control. The greatest loss reduction is obtained with the slot aligned along the duct. If the amplitude of membrane vibrations is not properly chosen, the total pressure loss reduction is not that large.

\section{CONCLUDING REMARKS}

A numerical study of parameters and separated flow structures in a spatial S-shaped diffuser was performed with the use of the RANS/URANS methods. A complex spatial nonstationary structure was obtained. A comprehensive study of using a generator of synthetic jets allowed obtaining the total pressure loss reduction by $45 \%$ with a membrane vibration frequency of $1 \mathrm{kHz}$.

\section{ACKNOWLEDGMENTS}

The work was supported by the Russian Foundation for Basic Research (project code 08-01-00308) and by the program for supporting the leading scientific schools of the Russian Federation (project code NSh-3876.2008.1).

\section{REFERENCES}

1. Konovalov, A.E., S. Yu. Krasheninnikov, D. A. Lubimov, D. E. Pudovikov, and V.A. Stepanov. 2009. Experimental and numerical singularity investigations of separated flow in subsonic ducts. Fluid Dynamics 44(4).

2. Wellborn, S. R., B. A. Reichret, and T. H. Okiishi. 1992. An experimental investigation of the flow in a diffusing S-duct. AIAA Paper No. 92-3622.

3. Zhang, W.-L., D. Knight, and D. Smith. 2000. Automated design of a threedimensional subsonic diffuser. J. Propul. Power 16(6):1132-40.

4. Vuillerme, A. L., S. Deck, and R. Chevrier. 2005. Numerical simulations of the flow inside an S-shaped intake diffuser. EUCASS.

5. Krasheninnikov, S. Yu., and D. E. Pudovikov. 2007. Induced flow and ascent of heavy particles when an air intake operates near a surface. Fluid Dynamics 42(4):654-65.

6. Krasheninnikov, S. Yu., and D. E. Pudovikov. 2007. Induced flow and large particles lift at air intake operation near ground surface. EUCASS.

7. Cannelle, F., and M. Amitay. 2005. Synthetic jets spatial evolution and transitory behavior. AIAA Paper No. 2005-102.

8. Holman, R., Y. Utturkar, R. Mittal, B. L. Smith, and L. Cattafesta. 2005. Formation criterion for synthetic jets. AIAA J. 43(10):2110-16. 